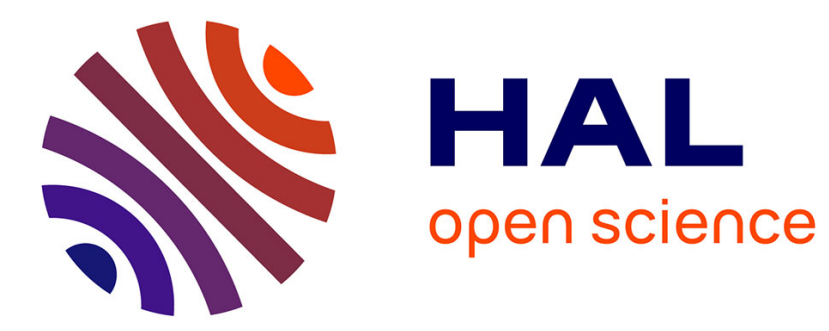

\title{
Bases and Transforms of Set Functions
}

\author{
Michel Grabisch
}

\section{To cite this version:}

Michel Grabisch. Bases and Transforms of Set Functions. S. Saminger-Platz and R. Mesiar. On Logical, Algebraic and Probabilistic Aspects of Fuzzy Set Theory, 2016, 10.1007/978-3-319-288086_13. hal-01302376

\section{HAL Id: hal-01302376 https://hal.science/hal-01302376}

Submitted on 14 Apr 2016

HAL is a multi-disciplinary open access archive for the deposit and dissemination of scientific research documents, whether they are published or not. The documents may come from teaching and research institutions in France or abroad, or from public or private research centers.
L'archive ouverte pluridisciplinaire HAL, est destinée au dépôt et à la diffusion de documents scientifiques de niveau recherche, publiés ou non, émanant des établissements d'enseignement et de recherche français ou étrangers, des laboratoires publics ou privés. 


\title{
Bases and transforms of set functions
}

\author{
Michel GRABISCH \\ Paris School of Economics, University of Paris I \\ 106-112, Bd de l'Hôpital, 75013 Paris, France \\ michel.grabisch@univ-paris1.fr
}

\begin{abstract}
The paper studies the vector space of set functions on a finite set $X$, which can be alternatively seen as pseudo-Boolean functions, and including as a special cases games. We present several bases (unanimity games, Walsh and parity functions) and make an emphasis on the Fourier transform. Then we establish the basic duality between bases and invertible linear transform (e.g., the Möbius transform, the Fourier transform and interaction transforms). We apply it to solve the well-known inverse problem in cooperative game theory (find all games with same Shapley value), and to find various equivalent expressions of the Choquet integral.
\end{abstract}

\section{Introduction}

Set functions on a finite set $X$ are of fundamental usage in many areas of discrete mathematics, e.g., cooperative game theory (Peleg and Sudhölter, 2003), combinatorial optimization (Fujishige, 2005), decision making (Grabisch and Labreuche, 2010), computer sciences (de Wolf, 2008), and more generally operations research (Hammer and Rudeanu, 1968), where in the latter domain, they are more often encountered under the form of pseudo-Boolean functions. Specific domains focus on specific subclasses of set functions, e.g., game theory uses set functions vanishing on the empty set (these are characteristic functions of transferable utility games, which are simply called "games"), decision theory needs games which are monotone with respect to inclusion (called capacities), while combinatorial optimization often deals with submodular games.

An interesting feature of set functions and games is that they form a vector space of dimension $2^{|X|}\left(2^{|X|-1}\right.$ for games). Most often, this feature is ignored, although clearly one can take advantage of the concepts and techniques of linear algebra when dealing with set functions and games. In particular the notion of basis is of importance. The best-known basis is perhaps the basis of unanimity games (this is the usual name given in game theory; they are closely related to the incidence functions in combinatorics, see Aigner (1979)), although each domain has its prefered bases. For example, in computer sciences mostly the basis of parity functions is used, essentially because the encoding of sets is done by $-1,+1$, rather than by 0,1 . The usage of a particular basis induces a particular representation of set functions, viewed as a transform, which is by definition linear and invertible. For example, the representation through the basis of unanimity 
games is the Möbius transform (or Möbius inverse), widely used in combinatorics and well known in decision making and game theory (known under the name of Harsanyi dividends (Harsanyi, 1963) in the latter domain), while the representation through parity functions is the Fourier transform, which has many applications in computer sciences.

As far as the author can see from the literature, the duality between bases and transforms (i.e., the representation of set functions into a given basis) has never been exploited nor even remarked. A systematic exploitation of this fact can lead to the discovery of new bases and transforms, as well as an easy solution to the so called inverse problem in game theory: find all games having the same Shapley value (and similar ones). Also, it permits to get several different expressions of linear operators on games or set functions, like the Choquet integral.

The aim of this paper is to bring a survey on the above mentioned issues. Section 2 gives a brief account on set functions and pseudo-Boolean functions. Section 3 describes the best-known bases (unanimity games, Walsh functions and parity functions). Section 4 is about the Fourier transform and its properties, while Section 5 explains the fundamental duality between bases and transforms, and gives many examples. Sections 6 and 7 are applications of this duality principle to the solution of the above mentioned inverse problem and to the finding of equivalent expressions of the Choquet integral.

\section{Set functions and pseudo-Boolean functions}

In the whole paper, we consider a finite universe $X$, with $|X|=n$. Occasionally, we will use the notation $[n]=\{1, \ldots, n\}$.

A set function on $X$ is a mapping $\xi: 2^{X} \rightarrow \mathbb{R}$. A game is a set function $v$ vanishing on the empty set: $v(\varnothing)=0$.

Clearly, the set $\mathbb{R}^{2^{X}}$ of set functions on $X$ is a $2^{n}$-dimensional vector space. We introduce on $\mathbb{R}^{2^{X}}$ the following scalar product:

$$
\left\langle\xi, \xi^{\prime}\right\rangle=\frac{1}{2^{n}} \sum_{S \subseteq X} \xi(S) \xi^{\prime}(S) .
$$

There is another vision of set functions, namely the pseudo-Boolean functions (Hammer and Rudeanu, 1968), noting that any subset $A$ of $X$ can be encoded by its characteristic function $1_{A}$. Formally, a pseudo-Boolean function is a mapping $f:\{0,1\}^{n} \rightarrow \mathbb{R}$. The equivalence between pseudo-Boolean functions and set functions can be seen through the coding function $1: 2^{X} \rightarrow\{0,1\}^{n}$ defined by $A \mapsto 1_{A}$, with $1_{A}(i)=1$ if and only if $i \in A$. Then

$$
\xi_{f}=f \circ 1, \quad f_{\xi}=\xi \circ 1^{-1}
$$

where $\xi_{f}$ denotes the set function associated to $f$, and $f_{\xi}$ is the pseudo-Boolean function associated to $\xi$. If follows that the set of pseudo-Boolean functions of $n$ variables is a $2^{n}$-dimensional vector space, with scalar product

$$
\left\langle f, f^{\prime}\right\rangle=\frac{1}{2^{n}} \sum_{x \in\{0,1\}^{n}} f(x) f^{\prime}(x) .
$$


Pseudo-Boolean functions were obtained from set functions by coding subsets by 1 and 0 . It is noteworhty that other encodings are possible, for example using $-1,1$ instead of 0,1 . We will come back to this encoding later and shows that it is quite useful.

\section{Bases of set functions and pseudo-Boolean func- tions}

\subsection{Unanimity games}

Perhaps the best known basis of $\mathbb{R}^{2^{X}}$ is the basis of the so-called unanimity games. For any nonempty subset $S \subseteq X$, the unanimity game centered on $S$ is the game defined by

$$
u_{S}(T)=\left\{\begin{array}{ll}
1, & \text { if } T \supseteq S \\
0, & \text { otherwise }
\end{array} .\right.
$$

Defining the set function $u_{\varnothing}(S)=1$ for every $S \subseteq X$, it is well known that $\left\{u_{S}\right\}_{S \in 2^{X}}$ is a basis for $\mathbb{R}^{2^{X}}$. It is also well known that the coordinates of $\xi$ in this basis are the Möbius transform coefficients:

$$
\xi=\sum_{S \in 2^{X}} m^{\xi}(S) u_{S}
$$

with

$$
m^{\xi}(S)=\sum_{T \subseteq S}(-1)^{|S \backslash T|} \xi(T) .
$$

The Möbius transform (or Möbius inverse) is a well-known tool in combinatorics since the work of Rota (1964) (see also Berge (1971), Aigner (1979), etc.).

A drawback of the basis of unanimity games is that it is not orthogonal w.r.t. the above scalar product, as it is easy to see even with $n=2$ :

$$
\begin{aligned}
\left\langle u_{\{1\}}, u_{\{2\}}\right\rangle & =\frac{1}{4}\left(u_{\{1\}}(\{1\}) u_{\{2\}}(\{1\})+u_{\{1\}}(\{2\}) u_{\{2\}}(\{2\})+u_{\{1\}}(\{1,2\}) u_{\{2\}}(\{1,2\})\right. \\
& =\frac{1}{4} \neq 0 .
\end{aligned}
$$

In the formalism of pseudo-Boolean functions, unanimity games $u_{T}$ correspond to monomials $\prod_{i \in T} x_{i}$. Hence we have

$$
f=\sum_{T \subseteq[n]} m^{f}(T) \prod_{i \in T} x_{i}
$$

where $m^{f}$ is the Möbius transform of $\xi_{f}$ (this slight abuse of notation should not be confusing).

\subsection{Walsh functions}

Another basis of pseudo-Boolean functions is the basis of Walsh functions, which are monomials defined by

$$
w_{T}(x)=\prod_{i \in T}\left(2 x_{i}-1\right) \quad\left(T \subseteq[n], x \in\{0,1\}^{n}\right)
$$


or, in set function notation:

$$
w_{T}(S)=(-1)^{|T \backslash S|} \quad\left(S, T \in 2^{X}\right) .
$$

It can be shown that the Walsh functions $\left\{w_{T}\right\}_{T \subseteq[n]}$ form an orthornomal basis of the pseudo-Boolean functions:

$$
\left\langle w_{S}, w_{T}\right\rangle=1 \text { iff } S=T \text {, and } 0 \text { otherwise. }
$$

It is important to note that letting $z_{i}=2 x_{i}-1 \in\{-1,+1\}$, the Walsh functions reduce to the monomials $\prod_{i \in T} z_{i}$ : hence the Walsh functions are obtained when subsets are encoded by $-1,1$ instead of 0,1 . We see that this simple change makes the basis orthonormal. It can be shown that the coordinates of a pseudo-Boolean function into this basis are given by

$$
f(x)=\sum_{T \subseteq[n]}\left(\sum_{S \supseteq T} \frac{m^{f}(S)}{2^{|S|}}\right) w_{T}(x)=\sum_{T \subseteq[n]} \frac{1}{2^{|T|}} I_{\mathrm{B}}^{f}(T) w_{T}(x)
$$

where $I_{\mathrm{B}}^{f}$ is the Banzhaf interaction transform defined in terms of the Möbius transform by

$$
I_{\mathrm{B}}^{f}(T)=\sum_{S \supseteq T}\left(\frac{1}{2}\right)^{|S \backslash T|} m^{f}(S) \quad\left(T \in 2^{X}\right)
$$

(this transform will be properly introduced later).

As a historical remark, we note that the original Walsh functions (Walsh, 1923) are rather different in their definition:

$$
W_{k}(x)=(-1)^{\sum_{j=0}^{\infty} k_{j} x_{j+1}} \quad\left(k \in \mathbb{N}_{0}, x \in[0,1]\right),
$$

with $k=k_{0}+k_{1} 2+k_{2} 2^{2}+\cdots k_{m} 2^{m}, k_{i} \in\{0,1\}$ for all $i$, and $x=x_{1} 2^{-1}+x_{2} 2^{-2}+x_{3} 2^{-3}+\cdots$, $x_{i} \in\{0,1\}$ for all $i$, the binary representations of $k$ and $x$. They form an orthonormal basis of the set of square integrable functions on $[0,1]$. The connection with our Walsh functions is that the latter have a discretized domain

$$
0, \frac{1}{2^{n}}, \frac{2}{2^{n}}, \frac{3}{2^{n}}, \ldots, \frac{2^{n}-1}{2^{n}}
$$

of $2^{n}$ points, corresponding to the $2^{n}$ subsets of $[n]$. More precisely, $w_{S}(x)$ corresponds to $W_{k}\left(x^{\prime}\right)$ such that $S$ and $k$ have same binary coding, and

$$
x_{1}^{\prime}=1-x_{1}, \ldots, x_{n}^{\prime}=1-x_{n}, \quad \text { and } x_{j}^{\prime}=0 \text { for } j>n .
$$

\subsection{Parity functions}

Another family of functions related to the Walsh functions are the parity functions. The parity function associated to $S \subseteq[n]$ is the function

$$
\chi_{S}(x)=(-1)^{1_{S} \cdot x}=(-1)^{\sum_{i \in S} x_{i}} \quad\left(x \in\{0,1\}^{n}\right) .
$$

Its name comes from the fact that it takes only values -1 and +1 , depending on whether there is an odd or even number of elements of coordinates of $x$ equal to 1 in $S$. It expression as a set function is

$$
\chi_{S}(T)=(-1)^{|S \cap T|} \quad\left(S, T \in 2^{X}\right) .
$$


Up to a recoding by $\varepsilon(1)=0$ and $\varepsilon(-1)=1$, the parity functions are the Walsh functions:

$$
w_{S}(z)=\prod_{i \in S} z_{i}=(-1)^{\sum_{i \in S} \varepsilon\left(z_{i}\right)}=\chi_{S}(\varepsilon(z)) \quad\left(z \in\{-1,1\}^{n}\right) .
$$

Consequently, they form another orthonormal basis of the vector space of pseudo-Boolean functions. The interest of parity functions is that they lead to the well-known Fourier transform, to which the next section is devoted.

\section{The Fourier transform}

In the basis of parity functions, it can be shown that any pseudo-Boolean function $f$ is expressed by

$$
f=\sum_{S \subseteq[n]} \widehat{f}(S) \chi_{S}
$$

where the coordinates of $f$ in this basis, denoted by $\widehat{f}(S)$, are given by

$$
\widehat{f}(S)=\left\langle f, \chi_{S}\right\rangle=\frac{1}{2^{n}} \sum_{x \in\{0,1\}^{n}}(-1)^{1_{S} \cdot x} f(x) \quad(S \subseteq[n])
$$

or, in terms of set functions,

$$
\widehat{\xi}(S)=\frac{1}{2^{n}} \sum_{T \subseteq[n]}(-1)^{|S \cap T|} \xi(T) \quad(S \subseteq[n]) .
$$

The set of coefficients $\widehat{f}(S), S \subseteq[n]$, is called the Fourier transform of $f$, and is widely used in computer sciences (see, e.g., a survey in de Wolf (2008), as well as O'Donnell (2007)).

We show now some properties of the Fourier transform, and to this end, we introduce some additional notions and notation. We may consider $x \in\{0,1\}^{n}$ as a random variable with uniform distribution. Then, the expected value and the variance of a pseudo-Boolean function $f$ are

$$
\begin{aligned}
\mathbb{E}[f] & =\frac{1}{2^{n}} \sum_{x \in\{0,1\}^{n}} f(x) \\
\operatorname{Var}[f] & =\mathbb{E}\left[(f-\mathbb{E}[f])^{2}\right]=\mathbb{E}\left[f^{2}\right]-\mathbb{E}^{2}[f] .
\end{aligned}
$$

The convolution product of two pseudo-Boolean functions $f, g$ is defined by

$$
(f * g)(x)=\frac{1}{2^{n}} \sum_{y \in\{0,1\}^{n}} f(x \oplus y) g(y) \quad\left(x \in\{0,1\}^{n}\right)
$$

where $\oplus$ denotes the coordinatewise binary addition:

$$
1 \oplus 1=0=0 \oplus 0, \quad 1 \oplus 0=0 \oplus 1=1 .
$$


In terms of set functions, we obtain the following expression:

$$
(f * g)(S)=\frac{1}{2^{n}} \sum_{T \subseteq[n]} f(S \Delta T) g(T) \quad\left(S \in 2^{X}\right) .
$$

The properties of the Fourier transform are gathered in the next theorem.

Theorem 1. Let $f, g$ be two pseudo-Boolean functions. The following holds.

(i) $f(\mathbf{0})=\sum_{S \subseteq[n]} \widehat{f}(S)$;

(ii) $\widehat{f}(\varnothing)=\mathbb{E}[f]$;

(iii) (Parseval's identity) $\|f\|^{2}=\sum_{S \subseteq[n]} \widehat{f}^{2}(S)$;

(iv) $\sum_{S \in 2^{[n]} \backslash\{\varnothing\}} \widehat{f}^{2}(S)=\operatorname{Var}[f]$

(v) $f$ is constant if and only if $\widehat{f}(S)=0$ for all $S \neq \varnothing$;

(vi) $\widehat{(f * g)}(S)=\widehat{f}(S) \widehat{g}(S)$ for all $S \in 2^{[n]}$.

The name "Fourier transform" comes from the work of Fourier on the representation of integrable functions. The Fourier transform of a function, viewed as a function of time, gives its frequency representation. Exactly the same results as those in Theorem 1 hold for the original Fourier transform, which explains its name in computer sciences. However, it must be noted that all the properties, except the last one on convolution, are direct consequences of the orthonormality of the basis. To the opinion of the author, this transform should be rather called the Walsh transform, since the definition of the Walsh function is an infinite version of the parity function used here, as a comparison of (3) and (4) reveals.

\section{Bases and linear transforms on set functions}

The previous sections have introduced various bases on set functions and pseudo-Boolean functions: the unanimity games, the Walsh functions and the parity functions. As a byproduct, two fundamental notions in combinatorics and computer sciences have appeared, namely the Möbius transform and the Fourier transform. The name "transform" intuitively means (in particular by reference to well-known transforms used in mathematics for the analysis of real-valued functions: the (original) Fourier transform and the Laplace transform, mainly) a representation in another domain, but equivalent to the original one, i.e., the main desirable characteristic of the transform is that it should be invertible.

Although it comes as an evidence from elementary considerations in linear algebra, there is a duality between bases and linear invertible transforms, which to the knowledge of the author, has never been exploited nor remarked. The aim of this section is to explain this duality and to apply it, first to the known bases and transforms so as to 
obtain new bases and transforms, and second to some well-known inverse problem and representation of integrals w.r.t. games.

We define a transform on the set of set functions on $X$ as a linear invertible mapping $\Psi: \mathbb{R}^{2^{X}} \rightarrow \mathbb{R}^{2^{X}}$, with $\xi \mapsto \Psi^{\xi}$. The following discussion is made easier if one consider set functions $\xi$ as row vectors and use matrix notation. To a basis $\left(b_{S}\right)_{S \in 2^{x}}$, we make correspond the matrix $B=\left[b_{S}\right]$ of row vectors $b_{S}$. Hence $\xi=\sum_{S \in 2_{X}} w_{S} b_{S}=w B$ is the expression of $\xi$ in this basis. The following lemma gives the exact equivalence between bases and transforms.

Lemma 1. (Faigle and Grabisch, 2014) For every basis $B$, there is a (unique) transform $\Psi$ such that for any $\xi \in \mathbb{R}^{2^{X}}$,

$$
\xi=\sum_{S \in 2^{X}} \Psi^{\xi}(S) b_{S}
$$

whose inverse $\Psi^{-1}$ is given by $\xi \mapsto\left(\Psi^{-1}\right)^{\xi}=\sum_{S \in 2^{x}} \xi(S) b_{S}=\xi B$.

Conversely, to any transform $\Psi$ corresponds a unique basis $B$ such that (7) holds, given by $b_{S}=\left(\Psi^{-1}\right)^{\delta_{S}}$.

In the above lemma, $\delta_{S}$ denotes the Dirac set function defined by

$$
\delta_{S}(T)=\left\{\begin{array}{ll}
1, & \text { if } S=T \\
0, & \text { otherwise }
\end{array} \quad\left(S \in 2^{X}\right) .\right.
$$

We apply this result on a number of commonly used bases and transforms.

(i) The Möbius transform is, as we have already noticed, related to the basis of unanimity games:

$$
\xi(S)=\sum_{T \in 2^{X}} m^{\xi}(T) u_{T}(S)=\sum_{T \subseteq S} m^{\xi}(T), \quad(S \subseteq X)
$$

with

$$
m^{\xi}(S)=\sum_{T \subseteq S}(-1)^{|S \backslash T|} \xi(T)
$$

(ii) The co-Möbius transform (Grabisch et al. (2000), a.k.a. commonality function (Shafer, 1976)) is defined by:

$$
\check{m}^{\xi}(S)=\sum_{T \supseteq X \backslash S}(-1)^{n-|T|} \xi(T)=\sum_{T \subseteq S}(-1)^{|T|} \xi(X \backslash T) \quad\left(S \in 2^{X}\right) .
$$

Its inverse relation is

$$
\xi(S)=\sum_{T \subseteq X \backslash S}(-1)^{|T|} \check{m}^{\xi}(T) .
$$

By Lemma 1, the associated basis is

$$
\check{u}_{T}(S)=\sum_{B \subseteq X \backslash S}(-1)^{|B|} \delta_{T}(B)=\left\{\begin{array}{cl}
(-1)^{|T|} & \text { if } S \cap T=\emptyset \\
0 & \text { otherwise }
\end{array}\right.
$$


(iii) The (Shapley) interaction transform (Grabisch, 1997) is defined by

$$
I^{\xi}(S)=\sum_{T \subseteq X \backslash S} \frac{(n-t-s) ! t !}{(n-s+1) !} \sum_{L \subseteq S}(-1)^{|S \backslash L|} \xi(T \cup L),
$$

and the inverse relation is given by

$$
\xi(S)=\sum_{K \subseteq X} \beta_{|S \cap K|}^{|K|} I^{\xi}(K)
$$

where

$$
\beta_{k}^{l}=\sum_{j=0}^{k}\left(\begin{array}{l}
k \\
j
\end{array}\right) B_{l-j} \quad(k \leq l),
$$

and $B_{0}, B_{1}, \ldots$ are the Bernoulli numbers. The first values of $\beta_{k}^{l}$ are given in Table 1.

$$
\begin{array}{l|rrrrr}
k \backslash l & 0 & 1 & 2 & 3 & 4 \\
\hline & & & & & \\
0 & 1 & -\frac{1}{2} & \frac{1}{6} & 0 & -\frac{1}{30} \\
1 & & \frac{1}{2} & -\frac{1}{3} & \frac{1}{6} & -\frac{1}{30} \\
2 & & & \frac{1}{6} & -\frac{1}{6} & \frac{2}{15} \\
3 & & & & 0 & -\frac{1}{30} \\
4 & & & & & -\frac{1}{30}
\end{array}
$$

Table 1: The coefficients $\beta_{k}^{l}$

The associated basis $\left\{b_{T}^{I}\right\}_{T \in 2^{X}}$ is

$$
b_{T}^{I}(S)=\beta_{|T \cap S|}^{|T|} \quad\left(S \in 2^{X}\right) .
$$

(iv) The Banzhaf interaction transform (Roubens, 1996) is defined by

$$
I_{\mathrm{B}}^{\xi}(S)=\left(\frac{1}{2}\right)^{n-s} \sum_{K \subseteq X}(-1)^{|S \backslash K|} \xi(K)
$$

with inverse relation

$$
\left(I_{\mathrm{B}}^{-1}\right)^{\xi}(S)=\sum_{K \subseteq X}\left(\frac{1}{2}\right)^{k}(-1)^{|K \backslash S|} \xi(K) .
$$

The associated basis $\left\{b_{T}^{I_{B}}\right\}_{T \in 2^{X}}$ is

$$
b_{T}^{I_{B}}(S)=\sum_{K \subseteq X}\left(\frac{1}{2}\right)^{k}(-1)^{|K \backslash S|} \delta_{T}(K)=\left(\frac{1}{2}\right)^{|T|}(-1)^{|T \backslash S|} .
$$


(v) The Fourier interaction transform: as it was already explained in Section 4, the Fourier transform, as given by (6), corresponds to the basis of parity functions, given by (5).

The relations between the Fourier transform and the Möbius and Banzhaf transforms are given as follows:

$$
\begin{gathered}
\widehat{\xi}(S)=(-1)^{|S|} \sum_{K \supseteq S} \frac{1}{2^{k}} m^{\xi}(K) . \\
\widehat{\xi}(S)=\left(\frac{-1}{2}\right)^{s} I_{\mathrm{B}}^{\xi}(S) .
\end{gathered}
$$

(vi) The Walsh basis: This basis is defined by (1). Let us recover the corresponding transform $\xi \mapsto W^{\xi}$ by using Lemma 1, which we already gave in (2). By Lemma 1, the inverse transform is immediate:

$$
\left(W^{-1}\right)^{\xi}(S)=\sum_{T \subseteq X} \xi(T)(-1)^{|T \backslash S|} .
$$

The direct transform is obtained by solving the linear system

$$
\xi(S)=\sum_{T \subseteq X} W^{\xi}(T)(-1)^{|T \backslash S|} \quad\left(S \in 2^{X}\right),
$$

or by simply noticing that $w_{T}(S)=2^{|T|} b_{T}^{I_{B}}(S)$, which from

$$
\xi(S)=\sum_{T \subseteq X} I_{B}^{\xi}(T) b_{T}^{I_{B}}(S)=\sum_{T \subseteq X} W^{\xi}(T) w_{T}(S)
$$

yields the components of $W^{\xi}$ as

$$
W^{\xi}(T)=\left(\frac{1}{2}\right)^{|T|} I_{B}^{\xi}(T) \quad\left(T \in 2^{X}\right) .
$$

We recover Formula (2). Note that the Fourier and Walsh bases are related as follows:

$$
\chi_{T}(S)=\chi_{S}(T)=(-1)^{|S \cap T|}=(-1)^{|S \backslash(X \backslash T)|}=w_{S}(X \backslash T) .
$$

Also, from (10), we find

$$
F^{\xi}(S)=(-1)^{s} W^{\xi}(S) \quad\left(S \in 2^{X}\right)
$$

(vii) The Yokote basis: (see Yokote et al. (2013); Yokote (2015)) it is a basis of the set of games, which is defined by

$$
b_{T}^{Y}(S)=\left\{\begin{array}{ll}
1, & \text { if }|S \cap T|=1 \\
0, & \text { otherwise }
\end{array} \quad\left(S \in 2^{X} \backslash \varnothing\right) .\right.
$$

Any game $v$ reads in this basis

$$
v=\sum_{T \in 2^{X} \backslash \varnothing} Y^{v}(T) b_{T}^{Y}
$$


where the coordinates $Y^{v}(S)$ define the Yokote transform $Y$. We give now $Y^{v}$ in terms of $m^{v}$ and $v$, as well as the inverse relations:

$$
\begin{aligned}
& m^{v}(S)=|S|(-1)^{|S|+1} \sum_{K \supseteq S} Y^{v}(K) \quad(\varnothing \neq S \subseteq X) . \\
& Y^{v}(S)=(-1)^{|S|+1} \sum_{K \supseteq S} \frac{1}{|K|} m^{v}(K) \quad(\varnothing \neq S \subseteq X) . \\
& Y^{v}(S)=\sum_{L \subseteq X}(-1)^{|S \cap L|+1} \frac{(n-s-l) !(s+l-1) !}{n !} v(L) .
\end{aligned}
$$

\begin{tabular}{|c|c|c|}
\hline & Transform & Basis \\
\hline Möbius & $m^{\xi}(S)=\sum_{T \subseteq S}(-1)^{|S \backslash T|} \xi(T)$ & $u_{T}(S)= \begin{cases}1, & \text { if } S \supseteq T \\
0, & \text { otherwise }\end{cases}$ \\
\hline $\begin{array}{l}\text { co- } \\
\text { Möbius }\end{array}$ & $\check{m}^{\xi}(S)=\sum_{T \supseteq X \backslash S}(-1)^{n-|T|} \xi(T)$ & $\check{u}_{T}(S)= \begin{cases}(-1)^{|T|}, & \text { if } S \cap T=\varnothing \\
0, & \text { otherwise }\end{cases}$ \\
\hline $\begin{array}{l}\text { conjugate } \\
\text { unanim- } \\
\text { ity } \\
\text { games }\end{array}$ & $\bar{U}^{\xi}(S)=(-1)^{|S|+1} \sum_{T \supseteq X \backslash S}(-1)^{n-|T|} \xi(T)$ & $\overline{u_{T}}(S)= \begin{cases}1, & \text { if } S \cap T \neq \varnothing \\
0, & \text { otherwise }\end{cases}$ \\
\hline $\begin{array}{l}\text { Shapley } \\
\text { interac- } \\
\text { tion }\end{array}$ & $I^{\xi}(S)=\sum_{K \subseteq X} \frac{|X \backslash(S \cup K)| !|K \backslash S| !}{(n-s+1) !}(-1)^{|S \backslash K|} \xi(K)$ & $b_{T}^{I}(S)=\beta_{|T \cap S|}^{|T|}$ \\
\hline $\begin{array}{l}\text { Banzhaf } \\
\text { interac- } \\
\text { tion }\end{array}$ & $I_{\mathrm{B}}^{\xi}(S)=\left(\frac{1}{2}\right)^{n-s} \sum_{K \subseteq X}(-1)^{|S \backslash K|} \xi(K)$ & $b_{T}^{I_{B}}(S)=\left(\frac{1}{2}\right)^{|T|}(-1)^{|T \backslash S|}$ \\
\hline Fourier & $\widehat{\xi}(S)=\frac{1}{2^{n}} \sum_{K \subseteq X}(-1)^{|S \cap K|} \xi(K)$ & $\chi_{T}(S)=(-1)^{|S \cap T|}$ \\
\hline Walsh & $W^{\xi}(S)=\frac{1}{2^{n}} \sum_{K \subseteq X}(-1)^{|S \backslash K|} \xi(K)$ & $w_{T}(S)=(-1)^{|T \backslash S|}$ \\
\hline $\begin{array}{l}\text { Yokote } \\
(S \neq \varnothing)\end{array}$ & $Y^{v}(S)=\sum_{L \subseteq X}(-1)^{|S \cap L|+1} \frac{(n-s-l) !(s+l-1) !}{n !} v(L)$ & $b_{T}^{Y}(S)= \begin{cases}1, & \text { if }|S \cap T|=1 \\
0, & \text { otherwise }\end{cases}$ \\
\hline
\end{tabular}

Table 5 summarizes the correspondence between bases and transforms.

Table 2: Correspondence between bases and transforms

\section{The inverse problem for linear values}

In cooperative game theory, a linear value is a linear mapping $\Phi: \mathbb{R}^{2^{X}} \rightarrow \mathbb{R}^{X}$ assigning to any game $v$ a $n$-dim vector $\Phi(v)$, representing a sharing of $v(X)$ among all players 
(elements of $X$ ). For this reason a value most often satisfies efficiency, in the sense that $\sum_{i \in X} \Phi_{i}(v)=v(X)$. The best-known values are the Shapley value Shapley (1953) and the Banzhaf value Banzhaf (1965). They are both linear and their definition amounts to considering the respective interaction transforms for singletons, i.e.:

$$
\Phi_{i}^{\mathrm{Sh}}(v)=I^{v}(\{i\}), \quad \Phi_{i}^{\mathrm{B}}(v)=I_{B}^{v}(\{i\}), \quad(i \in X) .
$$

Hence, the interaction and Banzhaf interaction transforms can be seen as extensions of these values.

The duality between transforms and bases permits to easily solve the so-called "inverse problem", well-known in game theory (see, e.g., Kleinberg and Weiss (1985), Dragan $(1991,2006)$ and Yokote et al. (2013)): given a game $v$ on $X$, find all games $v^{\prime}$ having the same Shapley value (or any other linear value: Banzhaf, egalitarian, etc.), i.e., $\Phi^{\mathrm{Sh}}(v)=$ $\Phi^{\mathrm{Sh}}\left(v^{\prime}\right)$.

Considering a linear value $\Phi$ and a game $v$, finding all games $v^{\prime}$ such that $\Phi(v)=\Phi\left(v^{\prime}\right)$ amounts by linearity to solving $\Phi\left(v-v^{\prime}\right)=0$, i.e., $v-v^{\prime} \in \operatorname{ker}(\Phi)$. Hence the solution of the inverse problem reduces to finding the kernel of the linear operator $\Phi$.

The kernel is easily found if there exists a transform $\Psi$ extending the linear value $\Phi$, exactly as the interaction transform extends the Shapley value (see (17)). Indeed, the kernel is just the space spanned by the vectors $f_{S}$ of the corresponding basis with $|S|>1$. We illustrate this method with the Shapley value. For any game $v$, its epxression in the basis induced by the interaction transform is:

$$
v=\sum_{S \in 2^{X}} I^{v}(S) b_{S}^{I}=\sum_{i \in X} \Phi_{i}^{S h}(v) b_{\{i\}}^{I}+\sum_{|S|>1} I^{v}(S) b_{S}^{I},
$$

which implies

$$
v \in \operatorname{ker}\left(\Phi^{\mathrm{Sh}}\right) \quad \Longleftrightarrow \quad v=\sum_{|S|>1} I^{v}(S) b_{S}^{I}
$$

i.e.,

$$
\operatorname{ker}\left(\Phi^{\mathrm{Sh}}\right)=\left\{\sum_{|S|>1} \lambda_{S} b_{S}^{I} \mid \lambda_{S} \in \mathbb{R}\right\} .
$$

In the case where $|X|=3$, we obtain, using Table 1 :

$$
\begin{aligned}
v(\emptyset) & =\lambda_{\emptyset}+\frac{1}{6}\left(\lambda_{12}+\lambda_{13}+\lambda_{23}\right) \\
v(1) & =\lambda_{\emptyset}-\frac{1}{3} \lambda_{12}-\frac{1}{3} \lambda_{13}+\frac{1}{6} \lambda_{23}+\frac{1}{6} \lambda_{123} \\
v(2) & =\lambda_{\emptyset}-\frac{1}{3} \lambda_{12}+\frac{1}{6} \lambda_{13}-\frac{1}{3} \lambda_{23}+\frac{1}{6} \lambda_{123} \\
v(3) & =\lambda_{\emptyset}+\frac{1}{6} \lambda_{12}-\frac{1}{3} \lambda_{13}-\frac{1}{3} \lambda_{23}+\frac{1}{6} \lambda_{123} \\
v(12) & =\lambda_{\emptyset}+\frac{1}{6} \lambda_{12}-\frac{1}{3} \lambda_{13}-\frac{1}{3} \lambda_{23}-\frac{1}{6} \lambda_{123} \\
v(13) & =\lambda_{\emptyset}-\frac{1}{3} \lambda_{12}+\frac{1}{6} \lambda_{13}-\frac{1}{3} \lambda_{23}-\frac{1}{6} \lambda_{123} \\
v(23) & =\lambda_{\emptyset}-\frac{1}{3} \lambda_{12}-\frac{1}{3} \lambda_{13}+\frac{1}{6} \lambda_{23}-\frac{1}{6} \lambda_{123} \\
v(123) & =\lambda_{\emptyset}+\frac{1}{6}\left(\lambda_{12}+\lambda_{13}+\lambda_{23}\right),
\end{aligned}
$$


where $\lambda_{S} \in \mathbb{R}$ for every $S \subseteq\{1,2,3\}$.

Let us give a second illustrative example with the Banzhaf value. Consider the following problem: Given a n-dim vector $y$, find all games $v$ s.t. $\Phi^{\mathrm{B}}(v)=y$. The set of solutions is simply the set of games of the form

$$
v=v_{y}+w, \text { with } w \in \operatorname{ker}\left(\Phi^{\mathrm{B}}\right),
$$

and $v_{y}$ is any game s.t. $\Phi^{\mathrm{B}}\left(v_{y}\right)=y$. Since the Banzhaf interaction transform generalizes the Banzhaf index, we have

$$
\operatorname{ker}\left(\Phi^{\mathrm{B}}\right)=\operatorname{Sp}\left\{b_{T}^{I_{\mathrm{B}}},|T|>1\right\}
$$

with $b_{T}^{I_{\mathrm{B}}}(S)=(1 / 2)^{|T|}(-1)^{|T \backslash S|}$, and Sp denotes the space spanned by the vectors. Now, $v_{y}$ can be obtained as the inverse transform of the game $w$ defined by $w(\{i\})=y_{i}$ for all $i \in X$, and $w(S)=0$ otherwise. This yields by (8):

$$
v_{y}(S)=\frac{1}{2}\left(\sum_{i \in S} y_{i}-\sum_{i \notin S} y_{i}\right) .
$$

In the case where there is no known transform which extends the linear value under consideration, a general method is given by Faigle and Grabisch (2014), consisting in the following steps:

(i) Select a basis $E=\left\{e_{1}, \ldots, e_{k}\right\}$ of the range $\Phi\left(\mathbb{R}^{2^{X}}\right)$.

(ii) Find set functions $b_{1}, \ldots, b_{k} \in \mathbb{R}^{2^{X}}$ such that $\Phi\left(b_{i}\right)=e_{i}, i=1, \ldots, k$.

(iii) Complete the independent set $\left\{b_{1}, \ldots, b_{k}\right\}$ to form a basis $B=\left\{b_{1}, \ldots, b_{2^{n}}\right\}$ of $\mathbb{R}^{2^{X}}$.

(iv) Compute the coordinates $\epsilon_{1}^{(j)}, \ldots, \epsilon_{k}^{(j)}$ of $\Phi\left(b_{j}\right)$ in $E$ for $j=k+1, \ldots, 2^{n}$.

(v) Compute $b_{j}^{\Phi}=b_{j}-\sum_{i=1}^{k} \epsilon_{i}^{(j)} b_{i}$ for $j=k+1, \ldots, 2^{n}$.

Finally, the basis of the kernel is $\left\{b_{k+1}^{\Phi}, \ldots, b_{2^{n}}^{\Phi}\right\}$.

\section{Alternative expressions of the Choquet integral}

A second application of Lemma 1 is to obtain various equivalent expressions of the Choquet integral. Let $v$ be a game on $X$, and $f: X \rightarrow \mathbb{R}_{+}$a real-valued nonnegative mapping. The Choquet integral (Choquet, 1953) of $f$ w.r.t. $v$ is defined by

$$
\int f \mathrm{~d} v=\int_{0}^{\infty} v(\{x \in X \mid f(x) \geq \alpha\}) \mathrm{d} \alpha
$$

which yields in the discrete case $(|X|=n)$ :

$$
\int f \mathrm{~d} v=\sum_{i=1}^{n}\left(f_{\sigma(i)}-f_{\sigma(i-1)}\right) v(\{\sigma(i), \ldots \sigma(n)\})
$$

where $\sigma$ is a permutation on $[n]$ such that $f_{\sigma(1)} \leq f_{\sigma(2)} \leq \cdots \leq f_{\sigma(n)}$, and $f_{\sigma(0)}=0$. 
Remark 1. Usually, the Choquet integral is defined w.r.t. a capacity, that is, a game which is monotone with respect to set inclusion. However, it is not possible to extend the definition to arbitrary set functions $\xi$, i.e., such that $\xi(\varnothing) \neq 0$. Indeed, if $\xi(\varnothing) \neq 0$, it is easily seen from (18) that the integral becomes unbounded.

The Choquet integral is positively homogeneous but not additive in general, i.e., $\int(f+g) \mathrm{d} v \neq \int f \mathrm{~d} v+\int g \mathrm{~d} v$. However, an interesting feature of this integral is that it is linear w.r.t. the game:

$$
\int f \mathrm{~d}\left(v+\alpha v^{\prime}\right)=\int f \mathrm{~d} v+\alpha \int f \mathrm{~d} v^{\prime}
$$

Expressing $v$ in some basis, it is then possible to get the expression of the Choquet integral w.r.t. the corresponding transform.

Let $\Psi$ be a linear invertible transform, and $\left\{b_{A}^{\Psi}\right\}_{A \in 2^{X}}$ be the corresponding basis of set functions. Due to Remark 1, one has to be careful because many bases are composed of set functions which are not games. Therefore, some adaptation is necessary. From a basis $\left\{b_{A}^{\Psi}\right\}_{A \in 2^{X}}$, we build a basis of games $\left\{b_{A}^{\prime \Psi}\right\}_{A \in 2^{X} \backslash\{\varnothing\}}$ as follows:

$$
b_{S}^{\prime \Psi}(T)= \begin{cases}b_{S}^{\Psi}(T), & \text { if } T \neq \varnothing \quad \\ 0, \text { otherwise } & \left(S \in 2^{X} \backslash\{\varnothing\}\right) .\end{cases}
$$

Then, from the linearity of the integral, for every $f \in \mathbb{R}^{X}$ and every game $v$,

$$
\int f \mathrm{~d} v=\int f \mathrm{~d}\left(\sum_{\varnothing \neq A \subseteq X} \Psi^{v}(A) b_{A}^{\prime \Psi}\right)=\sum_{\varnothing \neq A \subseteq X} \Psi^{v}(A) \int f \mathrm{~d} b_{A}^{\prime \Psi} .
$$

It is therefore sufficient to compute $\int f \mathrm{~d} b_{A}^{\prime \Psi}$ for every $A \subseteq X, A \neq \varnothing$. One obtains the following expressions, for the main bases:

- For the Möbius transform:

$$
\int f \mathrm{~d} u_{A}=\bigwedge_{i \in A} f_{i}
$$

- For the co-Möbius transform:

$$
\int f \mathrm{~d} \check{u}_{A}^{\prime}=(-1)^{|A|+1} \bigvee_{i \in A} f_{i}
$$

- For the Fourier transform:

$$
\int f \mathrm{~d} \chi_{A}^{\prime}=f_{\sigma(n)}+2 \sum_{j=1}^{|A|}(-1)^{j} f_{i_{j}}
$$

with $A=\left\{i_{1}, \ldots, i_{|A|}\right\}$ and $f_{i_{1}} \geqslant \cdots \geqslant f_{i_{|A|}}$. 
Equation (19) is well-known and was first proved by Chateauneuf and Jaffray (1989) (also by Walley (1981)), extending a result of Dempster (1967)).

There is no simple expression for the case of the interaction transform, although such an expression exists and has been obtained through a different method (Grabisch and Labreuche, 2002):

$$
\begin{aligned}
\int f \mathrm{~d} v= & \sum_{A \subseteq X}\left(\sum_{K \subseteq X \backslash A} B_{|K|} I^{v^{+}}(A \cup K)\right) \bigwedge_{i \in A} f_{i} \\
& +\sum_{\varnothing \neq A \in 2^{X}}(-1)^{|A|+1}\left(\sum_{K \subseteq X \backslash A} B_{|K|} I^{v^{-}}(A \cup K)\right) \bigvee_{i \in A} f_{i}
\end{aligned}
$$

where

$$
I^{v^{+}}(A)=\left\{\begin{array}{ll}
I^{v}(A), & \text { if } I^{v}(A)>0 \\
0, & \text { otherwise }
\end{array}, \quad I^{v^{-}}(A)=\left\{\begin{array}{ll}
I^{v}(A), & \text { if } I^{v}(A)<0 \\
0, & \text { otherwise }
\end{array} \quad\left(A \in 2^{X}\right) .\right.\right.
$$

\section{References}

M. Aigner. Combinatorial Theory. Springer Verlag, 1979.

J. Banzhaf. Weighted voting doesn't work: A mathematical analysis. Rutgers Law Review, 19:317-343, 1965.

C. Berge. Principles of Combinatorics. Academic Press, 1971.

A. Chateauneuf and J.-Y. Jaffray. Some characterizations of lower probabilities and other monotone capacities through the use of Möbius inversion. Mathematical Social Sciences, 17:263-283, 1989.

G. Choquet. Theory of capacities. Annales de l'Institut Fourier, 5:131-295, 1953.

R. de Wolf. A brief introduction to Fourier analysis on the Boolean cube. Theory of Computing Library Graduate Surveys, 1:1-20, 2008.

A. P. Dempster. Upper and lower probabilities induced by a multivalued mapping. Ann. Math. Statist., 38:325-339, 1967.

I. Dragan. The potential basis and the weighted Shapley value. Libertas Mathematica, 11:139-150, 1991.

I. Dragan. The least square values and the Shapley value for cooperative TU games. TOP, 14:61-73, 2006.

U. Faigle and M. Grabisch. Bases and linear transforms of cooperation systems. Working paper, 2014.

S. Fujishige. Submodular functions and optimization, volume 58 of Annals of Discrete Mathematics. Elsevier, Amsterdam, 2nd edition, 2005. 
M. Grabisch. k-order additive discrete fuzzy measures and their representation. Fuzzy Sets and Systems, 92:167-189, 1997.

M. Grabisch and Ch. Labreuche. The symmetric and asymmetric Choquet integrals on finite spaces for decision making. Statistical Papers, 43:37-52, 2002.

M. Grabisch and Ch. Labreuche. A decade of application of the Choquet and Sugeno integrals in multi-criteria decision aid. Annals of Operations Research, 175:247-286, 2010. doi: 10.1007/s10479-009-0655-8.

M. Grabisch, J.-L. Marichal, and M. Roubens. Equivalent representations of set functions. Mathematics of Operations Research, 25(2):157-178, 2000.

P. L. Hammer and S. Rudeanu. Boolean Methods in Operations Research and Related Areas. Springer, 1968.

J. C. Harsanyi. A simplified bargaining model for the $n$-person cooperative game. International Economic Review, 4:194-220, 1963.

N. L. Kleinberg and J. H. Weiss. Equivalent $n$-person games and the null space of the Shapley value. Mathematics of Operations Research, 10(2):233-243, 1985.

R. O'Donnell. Analysis of Boolean functions, draft 2.0, ch. 1-3. http://www.cs.cmu.edu/ odonnell11/boolean-analysis, 2007.

B. Peleg and P. Sudhölter. Introduction to the theory of cooperative games. Kluwer Academic Publisher, 2003.

G. C. Rota. On the foundations of combinatorial theory I. Theory of Möbius functions. Zeitschrift für Wahrscheinlichkeitstheorie und Verwandte Gebiete, 2:340-368, 1964.

M. Roubens. Interaction between criteria and definition of weights in MCDA problems. In 44th Meeting of the European Working Group "Multicriteria Aid for Decisions", Brussels, Belgium, October 1996.

G. Shafer. A Mathematical Theory of Evidence. Princeton Univ. Press, 1976.

L. S. Shapley. A value for $n$-person games. In H. W. Kuhn and A. W. Tucker, editors, Contributions to the Theory of Games, Vol. II, number 28 in Annals of Mathematics Studies, pages 307-317. Princeton University Press, 1953.

P. Walley. Coherent lower (and upper) probabilities. Technical Report 22, University of Warvick, Coventry, 1981.

J. Walsh. A closed set of normal orthogonal functions. American Journal of Mathematics, 45:5-24, 1923.

K. Yokote. Weak addition invariance and axiomatization of the weighted Shapley value. Int. J. of Game Theory, 44:275-293, 2015.

K. Yokote, Y. Funaki, and Y. Kamijo. Linear basis to the Shapley value. Technical report, Waseda Economic Working Paper Series, 2013. 